\title{
Persistent corneal epithelial defect responding to rebamipide ophthalmic solution in a patient with diabetes
}

This article was published in the following Dove Press journal:

International Medical Case Reports Journal

10 May 2016

Number of times this article has been viewed

\section{Yusuke Hayashi \\ Hiroshi Toshida \\ Yusuke Matsuzaki \\ Asaki Matsui \\ Toshihiko Ohta}

Department of Ophthalmology, Juntendo University Shizuoka

Hospital, Izunokuni, Shizuoka, Japan
Correspondence: Hiroshi Toshida

Department of Ophthalmology, Juntendo University Shizuoka Hospital, I I 29, Izunokuni City, Shizuoka 4I 0-2295, Japan

$\mathrm{Tel}+815594831 \mathrm{II}$

Fax +8I 55948335 I

Email toshida@juntendo.ac.jp
Objective: Rebamipide ophthalmic suspension was developed for the treatment of dry eyes and for other corneal diseases, promoting the secretion of both mucin in tear fluid and membraneassociated mucin, increasing the number of goblet cells, and restoring the barrier function of the corneal epithelium. We report a case of a persistent corneal epithelial defect in a patient with diabetes treated with topical application of rebamipide ophthalmic suspension.

Case presentation: A 73-year-old woman had a history of type 2 diabetes for 35 years and nonproliferative diabetic retinopathy for 23 years. She presented to our department with discharge and ophthalmalgia in the left eye. A corneal ulcer was detected, and culture of corneal scrapings was performed, with Staphylococcus aureus and Streptococcus canis being isolated. The infection was treated with levofloxacin eye drops and ofloxacin ophthalmic ointment based on the sensitivity profile of the isolate. However, a corneal epithelial defect persisted for approximately 2 months despite continuing treatment with $0.1 \%$ hyaluronic acid ophthalmic suspension and $0.3 \%$ ofloxacin eye ointment. Her hemoglobin A1c was $7.3 \%$. The persistent corneal epithelial defect showed improvement at 2 weeks after treatment with rebamipide unit dose $2 \%$ ophthalmic suspension, and it did not recur even when vitrectomy was subsequently performed for vitreous hemorrhage due to progression of diabetic retinopathy.

Conclusion: This is the first report about efficacy of rebamipide unit dose $2 \%$ ophthalmic suspension for presenting persistent corneal epithelial defect in a patient with diabetes. In the present case, the suggested mechanisms are the following: improving the corneal barrier function, stabilization of mucin on the keratoconjunctival epithelium, and improving the wettability and stability of the tear film, which resulted in the promotion of healing of the corneal epithelial defect in a short time period.

Keywords: rebamipide, diabetic keratitis, corneal epithelium, wound healing

\section{Introduction}

Ocular complications of diabetes include diabetic retinopathy, diabetic cataract, diabetic ocular motility disorder, neovascular glaucoma, secondary glaucoma, diabetic optic neuropathy, uveitis, and diabetic keratopathy. ${ }^{1,2}$ Among them, diabetic keratopathy is unlikely to be associated with irreversible visual impairment, but it can lead to persistent corneal epithelial damage that is difficult to treat. ${ }^{3}$ Moreover, there is the risk that corneal epithelial disorder could increase susceptibility to bacterial or fungal infections. ${ }^{4}$ In patients with diabetes, attention must be paid to the general increased risk of infection, which is not only a problem for the eyes.

Rebamipide was developed in the 1990s as an oral agent for the treatment of gastric ulcer and gastritis. ${ }^{5-7}$ In recent years, its mucosal-protective effect has also been applied 
to protect the keratoconjunctival epithelium, ${ }^{8,9}$ following the development of ophthalmic rebamipide products for the treatment of dry eyes. ${ }^{8-11}$ Rebamipide ophthalmic suspension has been reported to exhibit multiple pharmacological effects, such as promoting the secretion of both mucin in tear fluid and membrane-associated mucin, increasing the number of goblet cells, ${ }^{8,9}$ restoring the barrier function of the corneal epithelium, ${ }^{12,13}$ and anti-inflammatory activity. ${ }^{14}$ It has been reported that rebamipide ophthalmic suspension is effective not only for dry eyes but also for other corneal diseases such as Sjögren's syndrome, ${ }^{15}$ persistent corneal erosion, ${ }^{16}$ superficial limbic keratopathy, ${ }^{17}$ alkaline corneal injury, ${ }^{18}$ lid wiper epitheliopathy, ${ }^{19}$ and lagophathalmos. ${ }^{20}$ However, no reports have been published concerning the efficacy of rebamipide ophthalmic suspension for persistent corneal epithelial defects associated with diabetic keratopathy. Here, we report a case with persistent corneal epithelial defect after infectious corneal ulcer in a patient with diabetes, which resolved following topical application of $2 \%$ rebamipide ophthalmic suspension for $\sim 2$ weeks.

\section{Case presentation}

A 73-year-old woman presented to the Department of Ophthalmology of Juntendo University Shizuoka Hospital with a sudden onset of discharge and ophthalmalgia in the left eye. She had a history of type 2 diabetes for 35 years. Hemoglobin ( $\mathrm{Hb}$ ) A $1 \mathrm{c}$ had ranged between $7.2 \%$ and $8.0 \%$ in recent years, indicating inadequate glycemic control. Cataract surgery had been performed on both eyes $\sim 20$ years ago. She also had a history of nonproliferative diabetic retinopathy for $\sim 23$ years and had not undergone retinal photocoagulation. Her family history was unremarkable.

At the first visit to our hospital, a corneal ulcer was noted in the left eye with corneal opacity (Figure 1). Further, conjunctival injection and chemosis and/or conjunctivochalasis on the lower part of burbar conjunctiva were observed. Infectious corneal ulcer was then diagnosed. Funduscopy
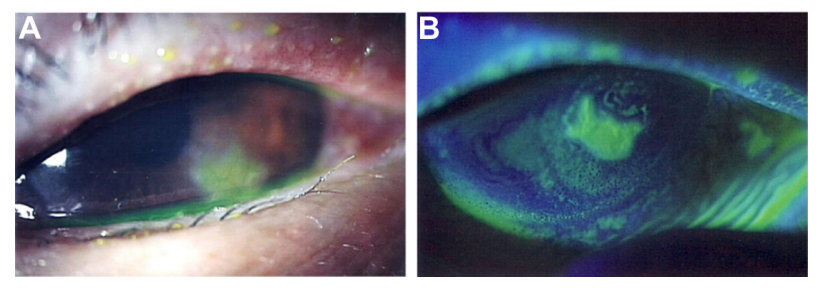

Figure I Slit lamp microscopic images of the left eye at the time of the initial presentation.

Notes: (A) A corneal ulcer with corneal opacity on the lower part of the cornea and conjunctival injection appeared. (B) Slit lamp image of the cornea with fluorescein staining showed defect of corneal epithelium. Chemosis and/or conjunctivochalasis on the lower part of burbar conjunctiva were shown. revealed mild retinal punctate hemorrhages in both eyes due to nonproliferative diabetic retinopathy. Corrected visual acuity was 14/20 and 16/20 in the right and left eyes, respectively. Topical application of $1.5 \%$ levofloxacin (LVFX) antimicrobial eye drops $\left(\mathrm{Cravit}^{\circledR}\right.$ ophthalmic solution $1.5 \%$; Santen Pharmaceutical Co. Ltd, Osaka, Japan) four times daily was commenced together with $0.3 \%$ ofloxacin ophthalmic ointment (Tarivid ${ }^{\circledR}$ ophthalmic ointment $0.3 \%$; Santen Pharmaceutical Co. Ltd) twice daily. Cefdinir capsules (Cefzon; Astellas Pharmaceutical Co. Ltd, Tokyo, Japan) were also administered orally (100 mg three times daily for 3 days). Staphylococcus aureus and Streptococcus canis were isolated by culture of corneal scrapings, and both of these bacteria were found to be sensitive to LVFX. At the first hospital visit, HbAlc was 7.3\%. Tear secretion was evaluated at $12 \mathrm{~mm}$ in the right eye and $11 \mathrm{~mm}$ in the left eye by Schirmer's tear test. Tear breakup time in both eyes was 4 seconds.

At 2 weeks after the start of treatment, there were no signs of infection, the corneal ulcer reduced, and her symptoms of eye discharge and the ophthalmalgia resolved. In contrast, clinically a lesion of corneal epithelial defect $\sim 1 \mathrm{~mm}$ in size was still noted. Even though additional administration of $0.1 \%$ hyaluronic ophthalmic suspension (Hyalein ${ }^{\circledR}$ ophthalmic solution 0.1\%; Santen Pharmaceutical Co. Ltd) six times daily, the corneal epithelial defect persisted (Figure 2). We diagnosed persistent corneal epithelial defect associated with diabetes as well as corneal hypoesthesia and diabetic keratitis. Vitreous hemorrhage occurred in the left eye $\sim 2$ months after the start of treatment, and visual acuity decreased from $16 / 20$ to $8 / 20$. The patient required vitrectomy, but it could not be done until the corneal epithelial defect healed. Accordingly, she was treated with $2 \%$ rebamipide ophthalmic suspension (Mucosta ophthalmic suspension unit dose 2\%; Otsuka Pharmaceutical Co. Ltd, Tokyo, Japan) four times

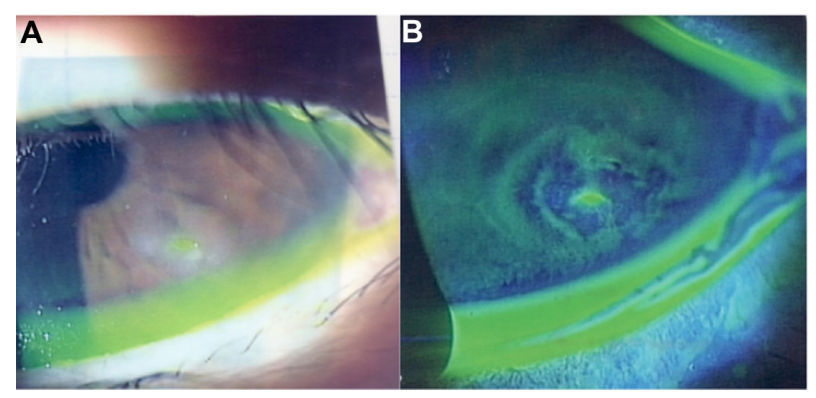

Figure 2 Slit lamp images from 2 months after the start of treatments.

Notes: (A) Despite the treatment, the corneal epithelial defect and opacity on the lower part of the cornea persisted. (B) Slit lamp image with fluorescein staining showed that the area of the corneal epithelium defect reduced. Chemosis and/or conjunctivochalasis were still shown. 
daily, and the corneal epithelial defect healed after 2 weeks. Vitrectomy was then performed, and administration of this drug was discontinued after vitrectomy, while LVFX eye drops and $0.1 \%$ betamethasone ophthalmic suspension were used. After surgery, any corneal epithelial defect did not recur after surgery (Figure 3), and her corrected visual acuity had recovered to $16 / 20$ at 3 months after surgery.

\section{Discussion}

In patients with a persistent corneal epithelial defect, a corneal epithelial lesion caused by trauma or surgery fails to heal. It is often difficult to treat, and patients may have underlying diseases such as diabetes, corneal neuropathy, or corneal epithelium adhesion factor disorder. ${ }^{21}$ A persistent corneal epithelial defect is generally treated by topical application of artificial tears, hyaluronic acid, and ophthalmic ointment, wearing a therapeutic soft contact lens, or topical application of serum. ${ }^{22,23}$ However, the defect is often refractory to these treatments. Use of fibronectin or peptide has also been investigated by some researchers, but these agents are not available yet clinically. ${ }^{21,24}$ The risk of infection is increased, particularly when the patient has diabetes. ${ }^{4}$ In the present patient, bacterial infection could have occurred due to a corneal epithelial disorder related to poor glycemic control, and a persistent corneal epithelial defect was noted after treatment of the infection. Diabetic keratopathy may be associated with neuropathic keratitis in patients with a persistent corneal epithelial defect, and dry eyes may occur secondary to a reflex decrease of tear secretion due to corneal hypoesthesia and/or secondary to reduced tear and mucin secretion due to efferent nerve dysfunction. ${ }^{25}$ Tear secretion

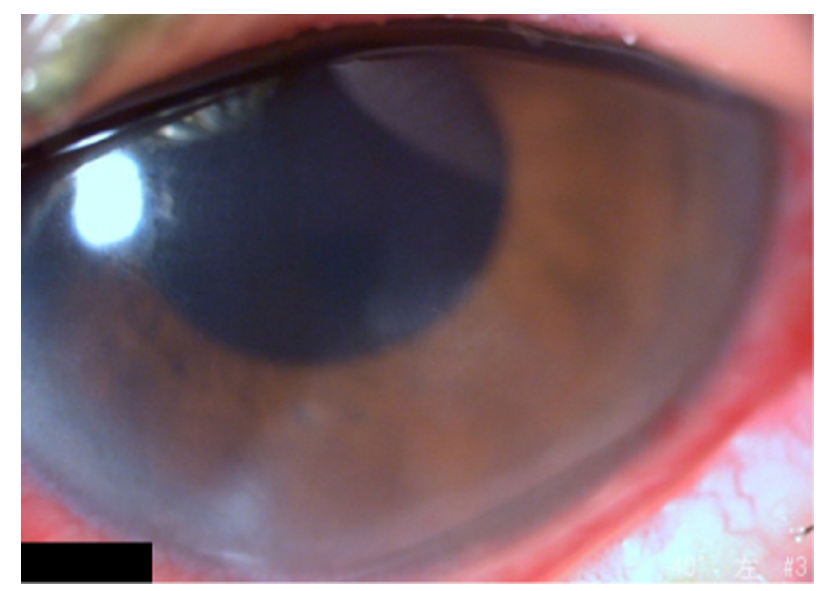

Figure 3 Slit lamp image from 3 weeks after the start of treatments with Mucosta ophthalmic suspension.

Notes: The corneal epithelial defect had then resolved, even though subepithelial opacity slightly remained. As this picture was taken after vitrectomy for treatment of vitreous hemorrhage, conjunctival hyperemia was shown. was not decreased in this patient, but there was a possibility of other neurological dysfunction. The development of diabetic keratopathy has been also suggested to be related to loss of nerve-derived trophic factors following a decrease in corneal sensation. Furthermore, there was another possibility of dysfunction of the tear meniscus and/or an ectopic tear meniscus by conjunctivochalasis or chemosis as shown in Figures 1 and 2. It might bring disturbance of stability of the tear film as described in the previous report ${ }^{26}$ and lead to persistent corneal epithelial defect.

In this patient, a persistent corneal epithelial defect that was refractory to standard treatment resolved after $\sim 2$ weeks of topical therapy with rebamipide ophthalmic suspension and did not recur when vitrectomy was performed subsequently. During the follow-up period, HbA1c ranged between $6.7 \%$ and $7.3 \%$, showing little fluctuation. The main pharmacological effects of rebamipide ophthalmic suspension include promoting the secretion of mucin in tears and membraneassociated mucin, increasing the number of goblet cells, and restoring the barrier function of the corneal epithelium by increasing the expression of tight junction proteins, as well as an anti-inflammatory effect. ${ }^{8-14}$ Such actions could have been exhibited in this patient with diabetic keratopathy. In brief, as a result of junctions between corneal epithelial cells being strengthened to improve corneal barrier function, mucin was stabilized on the corneal epithelium, and this improved the wettability and stability of the tear film, resulting in promotion of healing of the corneal epithelial defect. It has also been reported that inflammation occurs due to oxidative stress and increased expression of nuclear factor-kappa B in diabetic keratopathy. ${ }^{27}$ Rebamipide has been reported to have an anti-inflammatory effect and to inhibit the production of interleukin-6, interleukin- 8 , and tumor necrosis factor- $\alpha$ by human corneal epithelial cells, ${ }^{12-14}$ so these anti-inflammatory effects would also lead to improvement of persistent corneal epithelial defect.

\section{Conclusion}

Rebamipide ophthalmic suspension exhibited a beneficial effect on a presenting persistent corneal epithelial defect in a patient with diabetes relatively soon after the start of treatment.

\section{Acknowledgment}

The institutional review board at Juntendo University Shizuoka Hospital did not require the authors to obtain ethics approval and patient consent, following "Ethical Guidelines for Medical and Health Research Involving Human Subjects" 
established by the Ministry of Education, Culture, Sports, Science and Technology and the Ministry of Health, Labour and Welfare. ${ }^{28}$ Written informed consent was obtained from the patient before the patient received vitrectomy.

\section{Disclosure}

The authors report no conflicts of interest in this work.

\section{References}

1. Recchia FM, Connolly B, Benson WE. Ocular manifestations of diabetes. Curr Opin Ophthalmol. 1998;9(6):64-68.

2. Stanga PE, Boyd SR, Hamilton AM. Ocular manifestations of diabetes mellitus. Curr Opin Ophthalmol. 1999;10(6):483-489.

3. Kaji Y. Prevention of diabetic keratopathy. Br J Ophthalmol. 2005; 89(3):254-255.

4. DeMill DL, Hussain M, Pop-Busui R, Shtein RM. Ocular surface disease in patients with diabetic peripheral neuropathy. Br JOphthalmol. Epub 2015 Oct 23.

5. Uchida M, Tabusa F, Komatsu M, Morita S, Kanbe T, Nakagawa K. Studies on 2(1H)-quinolinone derivatives as gastric antiulcer active agents. 2-(4-Chlorobenzoylamino)-3-[2(1H)-quinolinon-4-yl] propionic acid and related compounds. Chem Pharm Bull (Tokyo). 1985;33(9):3775-3786.

6. Yamasaki K, Kanbe T, Chijiwa T, Ishiyama H, Morita S. Gastric mucosal protection by OPC-12759, a novel antiulcer compound, in the rat. Eur J Pharmacol. 1987;142(1):23-29.

7. Kleine A, Kluge S, Peskar BM. Stimulation of prostaglandin biosynthesis mediates gastroprotective effect of rebamipide in rats. Dig Dis Sci. 1993;38(8):1441-1449.

8. Urashima H, Okamoto T, Takeji Y, Shinohara H, Fujisawa S. Rebamipide increases the amount of mucin-like substances on the conjunctiva and cornea in the N-acetylcysteine-treated in vivo model. Cornea. 2004; 23(6):613-619.

9. Ríos JD, Shatos M, Urashima H, Tran H, Dartt DA. OPC-12759 increases proliferation of cultured rat conjunctival goblet cells. Cornea. 2006;25(5):573-581.

10. Kinoshita S, Awamura S, Oshiden K, et al; Rebamipide Ophthalmic Suspension Phase II Study Group. Rebamipide (OPC-12759) in the treatment of dry eye: a randomized, double-masked, multicenter, placebo-controlled phase II study. Ophthalmology. 2012;119(12): 2471-2478.

11. Kinoshita S, Oshiden K, Awamura S, et al; Rebamipide Ophthalmic Suspension Phase 3 Study Group. A randomized, multicenter phase 3 study comparing $2 \%$ rebamipide (OPC-12759) with $0.1 \%$ sodium hyaluronate in the treatment of dry eye. Ophthalmology. 2013;120(6): 1158-1165.

12. Tanaka H, Fukuda K, Ishida W, Harada Y, Sumi T, Fukushima A. Rebamipide increases barrier function and attenuates TNF $\alpha$-induced barrier disruption and cytokine expression in human corneal epithelial cells. Br J Ophthalmol. 2013;97(7):912-916.
13. Kimura K, Morita Y, Orita T, Haruta J, Takeji Y, Sonoda KH. Protection of human corneal epithelial cells from TNF- $\alpha$-induced disruption of barrier function by rebamipide. Invest Ophthalmol Vis Sci. 2013; 54(4):2752-2760.

14. Arakaki R, Eguchi H, Yamada A, et al. Anti-inflammatory effects of rebamipide eyedrop administration on ocular lesions in a murine model of primary Sjögren's syndrome. PLoS One. 2014;9(5):e98390.

15. Arimoto A, Kitagawa K, Mita N, Takahashi Y, Shibuya E, Sasaki H. Effect of rebamipide ophthalmic suspension on signs and symptoms of keratoconjunctivitis sicca in Sjögren syndrome patients with or without punctal occlusions. Cornea. 2014;33(8):806-811.

16. Kashima T, Akiyama H, Miura F, Kishi S. Resolution of persistent corneal erosion after administration of topical rebamipide. Clin Ophthalmol. 2012;6:1403-1406.

17. Takahashi Y, Ichinose A, Kakizaki H. Topical rebamipide treatment for superior limbic keratoconjunctivitis in patients with thyroid eye disease. Am J Ophthalmol. 2014;157(4):807-812.

18. Sasaki H, Kokubun T. Alkali burn treated with $2 \%$ rebamipide ophthalmic suspension: a case report. Case Rep Ophthalmol. 2014;5(3):380-385.

19. Itakura H, Kashima T, Itakura M, Akiyama H, Kishi S. Topical rebamipide improves lid wiper epitheliopathy. Clin Ophthalmol. 2013;7: 2137-2141.

20. Itakura M, Itakura H, Kashima $\mathrm{T}$, Akiyama H, Kishi S. Topical rebamipide improves the ocular surface in mild lagophthalmos. Clin Ophthalmol. 2013;7:1333-1338.

21. Wirostko B, Rafii M, Sullivan DA, Morelli J, Ding J. Novel therapy to treat corneal epithelial defects: a hypothesis with growth hormone. Ocul Surf. 2015;13(3):204-212.

22. Kanpolat A, Uçakhan OO. Therapeutic use of Focus Night and Day contact lenses. Cornea. 2003;22(8):726-734.

23. Tsubota K, Goto E, Shimmura S, Shimazaki J. Treatment of persistent corneal epithelial defect by autologous serum application. Ophthalmology. 1999;106(10):1984-1989.

24. Morita Y, Morishige N, Yamada N, Ohta M, Sonoda KH, Nishida T. Recovery of the corneal stroma associated with rapid reepithelialization induced by the fibronectin-derived peptide PHSRN in 2 cases of corneal perforation due to a persistent epithelial defect. Cornea. 2015;34(11):1504-1507.

25. Toshida H, Nguyen DH, Beuerman RW, Murakami A. Evaluation of novel dry eye model: preganglionic parasympathetic denervation in rabbit. Invest Ophthalmol Vis Sci. 2007;48(10):4468-4475.

26. Yokoi N, Inatomi T, Kinoshita S. Surgery of the conjunctiva. Dev Ophthalmol. 2008;41:138-158.

27. Kim J, Kim CS, Sohn E, Jeong IH, Kim H, Kim JS. Involvement of advanced glycation end products, oxidative stress and nuclear factorkappaB in the development of diabetic keratopathy. Graefes Arch Clin Exp Ophthalmol. 2011;249(4):529-536.

28. Ethical Guidelines for Medical and Health Research Involving Human Subjects. [Provisional Translation (as of March 2015)]. Ministry of Education, Culture, Sports, Science and Technology; Ministry of Health, Labour and Welfare; Japan. Available from: http://www.lifescience. mext.go.jp/files/pdf/n1500_01.pdf. Accessed April 21, 2016.
International Medical Case Reports Journal

\section{Publish your work in this journal}

The International Medical Case Reports Journal is an international, peer-reviewed open-access journal publishing original case reports from all medical specialties. Previously unpublished medical posters are also accepted relating to any area of clinical or preclinical science. Submissions should not normally exceed 2,000 words or

\section{Dovepress}

4 published pages including figures, diagrams and references. The manuscript management system is completely online and includes a very quick and fair peer-review system, which is all easy to use. Visit $\mathrm{http}: / /$ www.dovepress.com/testimonials.php to read real quotes from published authors. 